\title{
Chance Constrained Multi-Level Linear Programming Problem
}

\author{
Surapati Pramanik \\ Nandalal Ghosh B.T. College \\ P.O. Panpur, Narayanpur, Dist. \\ North 24 Parganas, India
}

\author{
Durga Banerjee \\ Ranaghat Yusuf Institution \\ P. O. Ranaghat,Dist. Nadia \\ India
}

\author{
B. C. Giri \\ Department of Mathematics \\ Jadavpur University
}

\begin{abstract}
In the paper, we present chance constrained multi-level linear programming problem. The right hand parameters and the coefficients of the constraints are considered as the random variables of known distribution function and the chance constraints are transformed into equivalent deterministic constraints. Membership function for each level objective function is constructed subject to the equivalent deterministic constraints. In the multi-level decision making situation, lower level decision makers may not be satisfied with the decision of higher level decision maker. To avoid this problem, each level decision maker provides relaxation in his/ her decision. Three FGP models are adopted to get the membership goals. Euclidean distance function is used to select the best FGP model offering the most satisfactory solution. Two numerical examples are solved to demonstrate the proposed approach.
\end{abstract}

\section{General Terms}

Decision Making, Linear Programming, Optimization.

\section{Keywords}

Multi-level programming, Fuzzy goal programming, Chance constrained programming.

\section{INTRODUCTION}

In multi-level programming (MLP), decision is sequential from upper level to successive lower levels. MLP is the extension of Stackleberg games [1]. Many pioneer researchers $[2,3,4,5,6,7,8,9,10,11,12$ and 13] have established different methodologies to solve MLP in both crisp and fuzzy environment. Anandalingam [1, 2] studied decentralized multi level programming problem in crisp environment. In fuzzy environment different models for MLP are studied by Lai [5], Shih et al. [8], Shih and Lee [9], Sakawa et el. [10], Sakawa et al. [11], Sinha [12, 13], Pramanik and Roy [14], Baky [6], and others. Pramanik and Roy [14] have established fuzzy goal programming (FGP) approach to MLP problems. In their approach, objective functions of all levels and the control vectors of upper level decision makers are fuzzily described. Membership functions for objective functions and controlled vectors are formulated. They considered deterministic system constraints. But in some real situations, system constraints or coefficients of objective function are not certain. Kumar and Pal [15] used FGP approach to solve chance constrained MLP. Chance constrained programming was studied by Charnes and Cooper [16]. In transportation, agriculture, different planning and policy schemes, economical field chance constraints are involved. Recently, three level quadratic programming with random rough coefficients in constraints has been described by Emam and Nasr [17]. In the first phase, they transfer rough nature constraints into equivalent crisp constraints using interval technique and in the second phase, they construct membership function and fuzzy model to obtain compromise solution. In 2015, Emam et al. [18] establish multi - level linear programming problem with random rough coefficients of objective functions. They convert the rough nature into equivalent crisp nature and form membership function to solve Tchebycheff problem till an optimal solution is obtained.

Pramanik and Dey [19] studied multi-objective quadratic programming problem based on Taylor series approximation. Pramanik and Banerjee [20] investigated fuzzy goal programming approach to chance constrained quadratic bilevel programming problem by extending the concept of Pramanik and Dey [19]. Pramanik et al. [21] also developed FGP models to solve chance constrained linear plus linear fractional bi-level programming problem. In the present paper, Pramanik and Banerjee's concept [20] has been extended to chance constrained multilevel linear programming problem (CCMLPP). Here, CCMLPP is considered with linear objective functions. Here, three types of chance constraints are considered. In first case, the coefficients of chance constraints are considered as random variables. The right hand parameters are taken as random variables in the second case. The aforesaid two cases are combined in the third case. The chance constraints are transformed into equivalent deterministic constraints by using known mean, variances of random variables and known satisfying probability levels. Each decision maker (DM) provides lower and upper limits on decision variables under his / her control for providing relaxation in his/her decision. Each objective function is fuzzily defined and membership function is constructed for each objective function. Using FGP technique, CCMLLPP is modelled.

The paper is designed as follows:

Section 2 is the formulation of CCMLLPP. Next section described three types of Chance constraints and the process of making deterministic constraints. Membership function for each objective function is developed in the section 4. In the next section, selection of bounds on the decision variables is made. Three FGP models are formulated in section 6. Process of determination of best compromise solution is described in section 7. A flowchart is given in the next section. In section 9 , numerical examples are solved and compared. In the next section conclusion and future studies are discussed. 


\section{FORMULATION OF CHANCE CONSTRAINED MULTI-LEVEL} LINEAR PROGRAMMING PROBLEM

The CCMLLPP can be presented as follows:

$$
\begin{aligned}
& \operatorname{Max}_{\mathrm{x}_{1}} \mathrm{z}_{1}(\mathrm{x})=\mathrm{a}_{11} \mathrm{x}_{1}+\mathrm{a}_{12} \mathrm{x}_{2}+\ldots+\mathrm{a}_{1 \mathrm{n}} \mathrm{x}_{\mathrm{n}} \\
& \operatorname{Max}_{\mathrm{x}_{2}} \mathrm{z}_{2}(\mathrm{x})=\mathrm{a}_{21} \mathrm{x}_{1}+\mathrm{a}_{22} \mathrm{x}_{2}+\ldots+\mathrm{a}_{2 \mathrm{n}} \mathrm{x}_{\mathrm{n}}
\end{aligned}
$$$$
\operatorname{Max}_{x_{n}} z_{n}(x)=a_{n 1} x_{1}+a_{n 2} x_{2}+\ldots+a_{n n} x_{n}
$$

subject to

$\operatorname{Pr}\left[\sum_{j=1}^{n} p_{i j} x_{j} \leq q_{i}\right] \geq 1-\delta_{i}, i=1,2, \ldots, r$

$x_{j} \geq 0, j=1,2, \ldots, n$

$\delta_{\mathrm{i}} \in(0,1), \mathrm{i}=1,2, \ldots, \mathrm{r}$

$\mathrm{p}_{\mathrm{ij}}$ andq $_{\mathrm{i}}$ are random variables following normal distribution and $\mathrm{X}=\left(\mathrm{X}_{1}, \mathrm{X}_{2}, \ldots, \mathrm{X}_{\mathrm{n}}\right)$. Here, we consider $\mathrm{n}$-level maximization type problem with constant coefficients of objective functions. We also assume that, the solution set is non-empty and bounded.

\section{CONVERSION OF CHANCE} CONSTRAINTS INTO EQUIVALENT DETERMINISTIC CONSTRAINTS

In this section, we discuss three possible cases [22]:

\subsection{Case - 1}

In this case $\mathrm{p}_{\mathrm{ij}}$ are considered as random variables for

$\mathrm{i}=1,2, \ldots, \mathrm{r}_{1}$.

Let $\mathrm{d}_{\mathrm{i}}=\sum_{\mathrm{j}=1}^{\mathrm{n}} \mathrm{p}_{\mathrm{ij}} \mathrm{x}_{\mathrm{j}}$ for $\mathrm{i}=1,2, \ldots, \mathrm{r}_{1}$.

Then the constraints reduces to

$$
\begin{aligned}
& \operatorname{Pr}\left[\mathrm{d}_{\mathrm{i}} \leq \mathrm{q}_{\mathrm{i}}\right] \geq 1-\delta_{\mathrm{i}} \quad \mathrm{i}=1,2, \ldots, \mathrm{r}_{1} \\
\Rightarrow & \operatorname{Pr}\left[\frac{\mathrm{d}_{\mathrm{i}}-\mathrm{E}\left(\mathrm{d}_{\mathrm{i}}\right)}{\sqrt{\operatorname{var}\left(\mathrm{d}_{\mathrm{i}}\right)}} \leq \frac{\mathrm{q}_{\mathrm{i}}-\mathrm{E}\left(\mathrm{d}_{\mathrm{i}}\right)}{\sqrt{\operatorname{var}\left(\mathrm{d}_{\mathrm{i}}\right)}}\right] \geq 1-\delta_{\mathrm{i}} \\
\Rightarrow & \mathrm{F}\left[\frac{\mathrm{q}_{\mathrm{i}}-\mathrm{E}\left(\mathrm{d}_{\mathrm{i}}\right)}{\sqrt{\operatorname{var}\left(\mathrm{d}_{\mathrm{i}}\right)}}\right] \geq 1-\delta_{\mathrm{i}} \\
\Rightarrow & 1-\mathrm{F}\left[-\frac{\mathrm{q}_{\mathrm{i}}-\mathrm{E}\left(\mathrm{d}_{\mathrm{i}}\right)}{\sqrt{\operatorname{var}\left(\mathrm{d}_{\mathrm{i}}\right)}}\right] \geq 1-\delta_{\mathrm{i}} \\
\Rightarrow & \mathrm{F}\left[-\frac{\mathrm{q}_{\mathrm{i}}-\mathrm{E}\left(\mathrm{d}_{\mathrm{i}}\right)}{\sqrt{\operatorname{var}\left(\mathrm{d}_{\mathrm{i}}\right)}}\right] \leq \delta_{\mathrm{i}} \\
\Rightarrow & {\left[-\frac{\mathrm{q}_{\mathrm{i}}-\mathrm{E}\left(\mathrm{d}_{\mathrm{i}}\right)}{\sqrt{\operatorname{var}\left(\mathrm{d}_{\mathrm{i}}\right)}}\right] \leq \mathrm{F}^{-1}\left(\delta_{\mathrm{i}}\right) }
\end{aligned}
$$

$$
\begin{aligned}
& \Rightarrow\left[-\mathrm{q}_{\mathrm{i}}+\mathrm{E}\left(\mathrm{d}_{\mathrm{i}}\right)\right] \leq \sqrt{\operatorname{var}_{\mathrm{i}}} \mathrm{F}^{-1}\left(\delta_{\mathrm{i}}\right) \\
& \Rightarrow \mathrm{q}_{\mathrm{i}} \geq \mathrm{E}\left(\mathrm{d}_{\mathrm{i}}\right)-\sqrt{\operatorname{var} \mathrm{d}_{\mathrm{i}}} \mathrm{F}^{-1}\left(\delta_{\mathrm{i}}\right)
\end{aligned}
$$

\subsection{Case - 2}

In this case, $\mathrm{q}_{\mathrm{i}}$ are random variables for $\mathrm{i}=\mathrm{r}_{1}+1, \ldots, \mathrm{r}_{2}$.

Then the constraints reduces to

$$
\begin{aligned}
& \operatorname{Pr}\left[d_{i} \leq q_{i}\right] \geq 1-\delta_{i} \\
\Rightarrow & \operatorname{Pr}\left[\frac{d_{i}-E\left(q_{i}\right)}{\sqrt{\operatorname{var}\left(q_{i}\right)}} \leq \frac{q_{i}-E\left(q_{i}\right)}{\sqrt{\operatorname{var}\left(q_{i}\right)}}\right] \geq 1-\delta_{i} \\
\Rightarrow & \delta_{i} \geq 1-\operatorname{Pr}\left[\frac{d_{i}-E\left(q_{i}\right)}{\sqrt{\operatorname{var}\left(q_{i}\right)}} \leq \frac{q_{i}-E\left(q_{i}\right)}{\sqrt{\operatorname{var}\left(q_{i}\right)}}\right] \\
\Rightarrow & \delta_{i} \geq \operatorname{Pr}\left[\frac{d_{i}-E\left(q_{i}\right)}{\sqrt{\operatorname{var}\left(q_{i}\right)}} \geq \frac{q_{i}-E\left(q_{i}\right)}{\sqrt{\operatorname{var}\left(q_{i}\right)}}\right] \\
\Rightarrow & \delta_{i} \geq F\left[\frac{d_{i}-E\left(q_{i}\right)}{\sqrt{\operatorname{var}\left(q_{i}\right)}}\right] \\
\Rightarrow & {\left[\frac{d_{i}-E\left(q_{i}\right)}{\sqrt{\operatorname{var}\left(q_{i}\right)}}\right] \leq F^{-1}\left(\delta_{i}\right) } \\
\Rightarrow & {\left[d_{i}-E\left(q_{i}\right)\right] \leq \sqrt{\operatorname{var} q_{i}} F^{-1}\left(\delta_{i}\right) } \\
\Rightarrow & d_{i} \leq E\left(q_{i}\right)+\sqrt{\operatorname{var} q_{i}} F^{-1}\left(\delta_{i}\right)
\end{aligned}
$$

\subsection{Case - 3}

In this case, $\mathrm{p}_{\mathrm{ij}}$ and $\mathrm{q}_{\mathrm{i}}$ both are considered as random variables. Let $g_{i}=\sum_{j=1}^{n} p_{i j} x_{j}-q_{i} \leq 0$ for $i=r_{2}+1, \ldots, r$.

Then the constraints reduces to

$$
\begin{aligned}
& \operatorname{Pr}\left[g_{\mathrm{i}} \leq 0\right] \geq 1-\delta_{\mathrm{i}} \quad \mathrm{i}=\mathrm{r}_{2}+1, \ldots, \mathrm{r} . \\
& \Rightarrow \operatorname{Pr}\left[\frac{\mathrm{g}_{\mathrm{i}}-\mathrm{E}\left(\mathrm{g}_{\mathrm{i}}\right)}{\sqrt{\operatorname{var}\left(\mathrm{g}_{\mathrm{i}}\right)}} \leq \frac{-\mathrm{E}\left(\mathrm{g}_{\mathrm{i}}\right)}{\sqrt{\operatorname{var}\left(\mathrm{g}_{\mathrm{i}}\right)}}\right] \geq 1-\delta_{\mathrm{i}} \\
& \Rightarrow \mathrm{F}\left[\frac{-\mathrm{E}\left(\mathrm{g}_{\mathrm{i}}\right)}{\sqrt{\operatorname{var}\left(\mathrm{g}_{\mathrm{i}}\right)}}\right] \geq 1-\delta_{\mathrm{i}} \\
& \Rightarrow 1-\mathrm{F}\left[\frac{\mathrm{E}\left(\mathrm{g}_{\mathrm{i}}\right)}{\sqrt{\operatorname{var}\left(\mathrm{g}_{\mathrm{i}}\right)}}\right] \geq 1-\delta_{\mathrm{i}} \\
& \Rightarrow \mathrm{F}\left[\frac{\mathrm{E}\left(\mathrm{g}_{\mathrm{i}}\right)}{\sqrt{\operatorname{var}\left(\mathrm{g}_{\mathrm{i}}\right)}}\right] \leq \delta_{\mathrm{i}} \\
& \Rightarrow \frac{\mathrm{E}\left(\mathrm{g}_{\mathrm{i}}\right)}{\sqrt{\operatorname{var}\left(\mathrm{g}_{\mathrm{i}}\right)}} \leq \mathrm{F}^{-1}\left(\delta_{\mathrm{i}}\right) \\
& \Rightarrow \mathrm{E}\left(\mathrm{g}_{\mathrm{i}}\right) \leq \mathrm{F}^{-1}\left(\delta_{\mathrm{i}}\right) \sqrt{\operatorname{var}\left(\mathrm{g}_{\mathrm{i}}\right)}
\end{aligned}
$$

Where 
$\operatorname{var}\left(\mathrm{g}_{\mathrm{i}}\right)=\sum_{\mathrm{j}=1}^{\mathrm{n}} \operatorname{var}\left(\mathrm{p}_{\mathrm{ij}}\right) \mathrm{x}_{\mathrm{j}}^{2}-\operatorname{var}\left(\mathrm{q}_{\mathrm{i}}\right)$

It is assumed that

$\operatorname{var}\left(\mathrm{g}_{\mathrm{i}}\right)=\sum_{\mathrm{j}=1}^{\mathrm{n}} \operatorname{var}\left(\mathrm{p}_{\mathrm{ij}}\right) \mathrm{x}_{\mathrm{j}}^{2}-\operatorname{var}\left(\mathrm{q}_{\mathrm{i}}\right) \geq 0$

Thus the proposed problem reduces

$\underset{x_{j}}{\operatorname{Max}_{j}} z_{j}(x)=a_{j 1} x_{1}+a_{j 2} x_{2}+\ldots+a_{j n} x_{n} \quad j=1,2, \ldots, n$

subject to the equivalent deterministic constraints

$\Rightarrow \mathrm{q}_{\mathrm{i}} \geq \mathrm{E}\left(\mathrm{d}_{\mathrm{i}}\right)-\sqrt{\operatorname{var} \mathrm{d}_{\mathrm{i}}} \mathrm{F}^{-1}\left(\delta_{\mathrm{i}}\right) \mathrm{i}=1,2, \ldots, \mathrm{r}_{1}$

$\Rightarrow \mathrm{d}_{\mathrm{i}} \leq \mathrm{E}\left(\mathrm{q}_{\mathrm{i}}\right)+\sqrt{\operatorname{var} \mathrm{q}_{\mathrm{i}}} \mathrm{F}^{-1}\left(\delta_{\mathrm{i}}\right) \quad \mathrm{i}=\mathrm{r}_{1}+1, \ldots, \mathrm{r}_{2}$

$\Rightarrow \mathrm{E}\left(\mathrm{g}_{\mathrm{i}}\right) \leq \mathrm{F}^{-1}\left(\delta_{\mathrm{i}}\right) \sqrt{\operatorname{var}\left(\mathrm{g}_{\mathrm{i}}\right)} \quad \mathrm{i}=\mathrm{r}_{2}+1, \ldots, \mathrm{r}$

where, $d_{i}=\sum_{j=1}^{n} p_{i j} x_{j}, g_{i}=\sum_{j=1}^{n} p_{i j} x_{j}-q_{i}, x_{j} \geq 0, \delta_{i} \in(0,1) \forall i$

Here, $F($.$) and \mathrm{F}^{-1}$ (.) represent the distribution function and inverse of the distribution function of standard normal variable respectively. Let us denote the system constraints (3), (5), (6), (7) by T.

\section{DEVELOPMENT OF MEMBERSHIP FUNCTION}

Each decision maker maximizes his/her objective function subject to the system constraints individually and considers the maximum value of the objective function as aspiration level.

Let $\underset{\mathrm{x}_{\mathrm{j}}}{\operatorname{Max}} \mathrm{z}_{\mathrm{j}}(\mathrm{x})=\mathrm{a}_{\mathrm{j} 1} \mathrm{x}_{1}+\mathrm{a}_{\mathrm{j} 2} \mathrm{x}_{2}+\ldots+\mathrm{a}_{\mathrm{jn}} \mathrm{x}_{\mathrm{n}}=\mathrm{z}_{\mathrm{j}}^{\mathrm{Max}}, \mathrm{x} \in \mathrm{T}$

$\mathrm{j}=1,2, \ldots, \mathrm{n}$ and the maximum solution point for the $\mathrm{j}$-th objective function is

$\mathrm{x}_{\mathrm{j}}^{\mathrm{Max}}=\left(\mathrm{X}_{\mathrm{j} 1}^{\mathrm{Max}}, \mathrm{x}_{\mathrm{j} 2}^{\mathrm{Max}}, \ldots, \mathrm{X}_{\mathrm{j} n}^{\mathrm{Max}}\right)$ i.e.

$\underset{x_{j}}{\operatorname{Max}} z_{j}(x)=a_{j 1} x_{1}+a_{j 2} x_{2}+\ldots+a_{j n} x_{n}=z_{j}^{M a x}=z_{j}\left(x_{j}^{\operatorname{Max}}\right)$

Then the fuzzy goal appears as:

$\mathrm{z}_{\mathrm{j}}(\mathrm{x}) \gtrsim \mathrm{z}_{\mathrm{j}}^{\mathrm{Max}}$ for $\mathrm{j}=1,2, \ldots, \mathrm{n}$

Let us now calculate the minimum value of each objective functions to select the lower tolerance limit of each objective function.

Let $\quad \operatorname{Min}_{\mathrm{x}_{\mathrm{j}}} \mathrm{z}_{\mathrm{j}}(\mathrm{x})=\mathrm{z}_{\mathrm{j}}^{\mathrm{Min}}, \mathrm{x} \in \mathrm{T}, \mathrm{j}=1,2, \ldots, \mathrm{n}$

The membership functions for fuzzy goals defined in (9) are

$\mu_{j}\left(z_{j}(x)\right)=\left(\begin{array}{lc}1, & z_{j} \geq z_{j}^{\operatorname{Max}} \\ \frac{z_{j}-z_{j}^{\text {Min }}}{z_{j}^{M a x}-z_{j}^{M i n}}, z_{j}^{\text {Min }} \leq z_{j} \leq z_{j}^{\text {Max }} \\ 0, & z_{j} \leq z_{j}^{\text {Min }}\end{array}\right\rangle$

$\mathrm{j}=1,2, \ldots, \mathrm{n}$

Now, the DM tries to maximize $\mu_{\mathrm{j}}\left(\mathrm{z}_{\mathrm{j}}(\mathrm{x})\right)$ such that $\mathrm{x} \in \mathrm{T}$.

\section{SELECTION OF BOUNDS ON THE DECISION VARIABLES}

It is obvious that each DM intends to maximize his/her objective function. But in MLPP, the decision is made sequentially from upper level to subsequent levels. To get overall satisfaction, cooperation between DMs is necessary. It is preferable to provide some bounds on decision variables. The lower and the upper bound of the decision variables are provided as follows:

$x_{j}^{*}-r_{j}^{-} \leq x_{j} \leq x_{j}^{*}+r_{j}^{+}, \quad j=1,2, \ldots, n$

$\mathrm{X}_{\mathrm{j}}^{*}$ is the individual best solution of the membership function $\mu_{\mathrm{j}}\left(\mathrm{z}_{\mathrm{j}}(\mathrm{x})\right)$ and $\mathrm{r}_{\mathrm{j}}^{-}, \mathrm{r}_{\mathrm{j}}^{+}$are positive quantity, may or may not be equal.

\section{FGP MODEL FORMULATION}

Following Pramanik and Banerjee [20] model, the present problem can be written as:

$\mu_{\mathrm{j}}\left(\mathrm{z}_{\mathrm{j}}(\mathrm{x})\right)+\mathrm{d}_{\mathrm{j}}^{-}=1 \mathrm{j}=1,2, \ldots, \mathrm{n}$

such that $\mathrm{x} \in \mathrm{T} . \mathrm{d}_{\mathrm{j}}^{-}$is under deviational variables and

non - negative quantity. Three FGP models are formulated to solve the problem.

\subsection{FGP Model - 1}

$\operatorname{Min} \alpha$

$\mu_{\mathrm{j}}\left(\mathrm{z}_{\mathrm{j}}(\mathrm{x})\right)+\mathrm{d}_{\mathrm{j}}^{-}=1$

$x_{j}^{*}-r_{j}^{-} \leq x_{j} \leq x_{j}^{*}+r_{j}^{+}$,

$\alpha \geq \mathrm{d}_{\mathrm{j}}^{-}$

$0 \leq \mathrm{d}_{\mathrm{j}} \leq 1 \quad$ and $\mathrm{x} \in \mathrm{T}, \mathrm{j}=1,2, \ldots, \mathrm{n}$

\subsection{FGP Model - 2}

$\operatorname{Min} \beta=\sum_{\mathrm{j}=1}^{\mathrm{n}} \mathrm{d}_{\mathrm{j}}^{-}$

$\mu_{\mathrm{j}}\left(\mathrm{z}_{\mathrm{j}}(\mathrm{x})\right)+\mathrm{d}_{\mathrm{j}}^{-}=1$

$\mathrm{x}_{\mathrm{j}}^{*}-\mathrm{r}_{\mathrm{j}}^{-} \leq \mathrm{x}_{\mathrm{j}} \leq \mathrm{x}_{\mathrm{j}}^{*}+\mathrm{r}_{\mathrm{j}}^{+}$,

$0 \leq \mathrm{d}_{\mathrm{j}}^{-} \leq 1 \quad$ and $\mathrm{x} \in \mathrm{T}, \mathrm{j}=1,2, \ldots, \mathrm{n}$

\subsection{FGP Model - 3}

$\operatorname{Min} \gamma=\sum_{j=1}^{n} \omega_{j} d_{j}$

$\mu_{\mathrm{j}}\left(\mathrm{z}_{\mathrm{j}}(\mathrm{x})\right)+\mathrm{d}_{\mathrm{j}}^{-}=1$,

$\mathrm{x}_{\mathrm{j}}^{*}-\mathrm{r}_{\mathrm{j}}^{-} \leq \mathrm{x}_{\mathrm{j}} \leq \mathrm{x}_{\mathrm{j}}^{*}+\mathrm{r}_{\mathrm{j}}^{+}, \quad 0 \leq \mathrm{d}_{\mathrm{j}}^{-} \leq 1 \quad$ and

$\mathrm{x} \in \mathrm{T}, \omega_{\mathrm{j}}=\frac{1}{\mathrm{z}_{\mathrm{j}}^{\text {Max }}-\mathrm{z}_{\mathrm{j}}^{\text {Min }}}, \mathrm{j}=1,2, \ldots, \mathrm{n}$ 


\section{DETERMINATION OF THE BEST COMPROMISE SOLUTION}

Here three FGP models are considered. Generally, it is expected that three models offer three different solutions. Euclidean distance function [20, 23 and 24] is used to identify which model provides better solution than other two models. Since in FGP models, the maximum value of membership function is assumed to be one and the deviation of optimal value of membership function from one is minimum. Using Euclidean distance function we try to find out the model which gives minimum deviation or distance. The Euclidean distance function is defined as follows:

$$
\mathrm{D}_{2}=\left[\sum_{\mathrm{j}=1}^{\mathrm{n}}\left\{1-\mu_{\mathrm{j}}\left(\mathrm{z}_{\mathrm{j}}(\mathrm{x})\right)\right\}^{2}\right]^{1 / 2}
$$

The solution having minimum value of $\mathrm{D}$ reflects the best compromise solution.

\section{SUMMARIZATION OF PROPOSED METHOD}

By the following steps, we describe our proposed method

Step -1 First chance constraints are converted into equivalent deterministic constraints.

Step - 2 Calculate individual best and worst solution for each

objective function subject to the deterministic constraints and membership function is formed for each objective function.

Step - 3 Each level DM provides his/her choice on the bounds of the decision variables.

Step -4 Three FGP models are formulated and solved.

Step - 5 Determine Euclidean distance functions for each model to select the best solution. The solution with minimum distance is considered as the best compromise solution.

\section{NUMERICAL EXAMPLES}

\subsection{Example - 1}

Consider the following multi-level (tri-level) linear programming problem with chance constraints.

$$
\begin{aligned}
& \underset{x_{1}}{\operatorname{Max}} z_{1}(x)=5 x_{1}+3 x_{2}+2 x_{3} \\
& \underset{x_{2}}{\operatorname{Max}} z_{2}(x)=6 x_{1}+12 x_{2}-x_{3} \\
& \operatorname{Max}_{x_{3}} z_{3}(x)=x_{1}+2 x_{2}+7 x_{3} \\
& \operatorname{Pr}\left[p_{11} x_{1}+p_{12} x_{2}+p_{13} x_{3} \leq 7\right] \geq 1-\delta_{1} \\
& \operatorname{Pr}\left[p_{21} x_{1}+p_{22} x_{2}+p_{23} x_{3} \leq q_{2}\right] \geq 1-\delta_{2} \\
& \operatorname{Pr}\left[2 x_{1}+x_{2}-x_{3} \leq q_{3}\right] \geq 1-\delta_{3}
\end{aligned}
$$

Here, $\delta_{1}=0.02, \mathrm{E}\left(\mathrm{p}_{11}\right)=2, \operatorname{var}\left(\mathrm{p}_{11}\right)=1, \mathrm{E}\left(\mathrm{p}_{12}\right)=2$,

$\operatorname{var}\left(\mathrm{p}_{12}\right)=2, \mathrm{E}\left(\mathrm{p}_{13}\right)=5, \operatorname{var}\left(\mathrm{p}_{13}\right)=4, \delta_{2}=0.03$,

$\mathrm{E}\left(\mathrm{p}_{21}\right)=7, \operatorname{var}\left(\mathrm{p}_{21}\right)=8, \mathrm{E}\left(\mathrm{p}_{22}\right)=12, \operatorname{var}\left(\mathrm{p}_{22}\right)=6, \mathrm{E}($ $\left.\mathrm{p}_{23}\right)=3, \operatorname{var}\left(\mathrm{p}_{23}\right)=9, \mathrm{E}\left(\mathrm{q}_{2}\right)=8, \operatorname{var}\left(\mathrm{q}_{2}\right)=2, \delta_{3}=0.01$, $\mathrm{E}\left(\mathrm{q}_{3}\right)=6, \operatorname{var}\left(\mathrm{q}_{3}\right)=4$.
Following (5), (6), (7) the above chance constraints become

$$
\begin{aligned}
& 2 \mathrm{x}_{1}+2 \mathrm{x}_{2}+5 \mathrm{x}_{3}+2.055 \sqrt{\mathrm{x}_{1}^{2}+2 \mathrm{x}_{2}^{2}+4 \mathrm{x}_{3}^{2}} \leq 7 \\
& 7 \mathrm{x}_{1}+12 \mathrm{x}_{2}+3 \mathrm{x}_{3}+1.885 \sqrt{8 \mathrm{x}_{1}^{2}+6 \mathrm{x}_{2}^{2}+9 \mathrm{x}_{3}^{2}-2} \leq 8 \\
& 2 \mathrm{x}_{1}+\mathrm{x}_{2}-\mathrm{x}_{3} \leq 1.35
\end{aligned}
$$

Assume that, $8 x_{1}^{2}+6 x_{2}^{2}+9 x_{3}^{2}-2 \geq 0$

The individual maximum and minimum value of each objective function subject to the equivalent deterministic constraints are obtained as follows:

Table 1. Max and Min values of objective functions

\begin{tabular}{|l|l|l|}
\hline $\mathrm{z}_{1}^{\mathrm{Max}}=3.778$ & $\mathrm{z}_{2}^{\mathrm{Max}}=7.577$ & $\mathrm{z}_{3}^{\mathrm{Max}}=5.428$ \\
\hline $\mathrm{z}_{1}^{\mathrm{Min}}=0.943$ & $\mathrm{z}_{2}^{\mathrm{Min}}=-0.768$ & $\mathrm{z}_{3}^{\mathrm{Min}}=0.5$ \\
\hline \\
Then the fuzzy goals appear as $\mathrm{z}_{1}(\mathrm{x})>3.778$,
\end{tabular}

$\mathrm{z}_{2}(\mathrm{x}) \gtrsim 7.577, \mathrm{z}_{3}(\mathrm{x}) \gtrsim 5.428$

The tolerance ranges of decision variables are expressed as follows:

$$
0.2 \leq \mathrm{x}_{1} \leq 0.8,0.2 \leq \mathrm{x}_{2} \leq 0.6,0.3 \leq \mathrm{x}_{3} \leq 0.8 .
$$

The three membership functions are constructed as follows:

$\mu_{1}\left(z_{1}(x)\right)=\left\langle\begin{array}{l}1, \quad z_{1} \geq 3.778 \\ \frac{z_{1}-0.943}{3.778-0.943}, 0.943 \leq z_{1} \leq 3.778 \\ 0, \quad z_{1} \leq 0.943\end{array}\right\rangle$

$\mu_{2}\left(z_{2}(x)\right)=\left(\begin{array}{l}1, \quad z_{2} \geq 7.577 \\ \frac{z_{2}+0.768}{7.577+0.768},-0.768 \leq z_{2} \leq 7.577 \\ 0, \quad z_{2} \leq-0.768\end{array}\right\rangle$

$\mu_{3}\left(z_{3}(x)\right)=\left\langle\begin{array}{lc}1, & z_{3} \geq 5.428 \\ \frac{z_{3}-0.5}{5.428-0.5}, & 0.5 \leq z_{3} \leq 5.428 \\ 0, & z_{3} \leq 0.5\end{array}\right\rangle$

The obtained solutions based on proposed three FGP models (14), (15) and (16) are presented in the table 2. 
Table 2. Result obtained from three FGP models

\begin{tabular}{|l|l|l|}
\hline Model-1 & Model-2 & Model-3 \\
\hline $\mathrm{x}_{1}=0.3737$ & $\mathrm{x}_{1}=0.4538$ & $\mathrm{x}_{1}=0.4934$ \\
\hline $\mathrm{x}_{2}=0.2120$ & $\mathrm{x}_{2}=0.2$ & $\mathrm{x}_{2}=0.2$ \\
\hline $\mathrm{x}_{3}=0.3929$ & $\mathrm{x}_{3}=0.2944$ & $\mathrm{x}_{3}=0.2196$ \\
\hline $\mathrm{z}_{1}=3.2903$ & $\mathrm{z}_{1}=3.3578$ & $\mathrm{z}_{1}=3.5062$ \\
\hline $\mathrm{z}_{2}=4.3933$ & $\mathrm{z}_{2}=4.8284$ & $\mathrm{z}_{2}=5.1408$ \\
\hline $\mathrm{z}_{3}=3.548$ & $\mathrm{z}_{3}=2.9146$ & $\mathrm{z}_{3}=2.4306$ \\
\hline$\mu_{\mathrm{z}_{1}}=0.6184$ & $\mu_{\mathrm{z}_{1}}=0.7494$ & $\mu_{\mathrm{z}_{1}}=0.7873$ \\
\hline$\mu_{\mathrm{z}_{2}}=0.6185$ & $\mu_{\mathrm{z}_{2}}=0.6706$ & $\mu_{\mathrm{z}_{2}}=0.7081$ \\
\hline$\mu_{\mathrm{z}_{3}}=0.6185$ & $\mu_{\mathrm{z}_{3}}=0.48997$ & $\mu_{\mathrm{z}_{3}}=0.3918$ \\
\hline $\mathrm{D}=0.6608$ & $\mathrm{D}=0.6568$ & $\mathrm{D}=0.7074$ \\
\hline
\end{tabular}

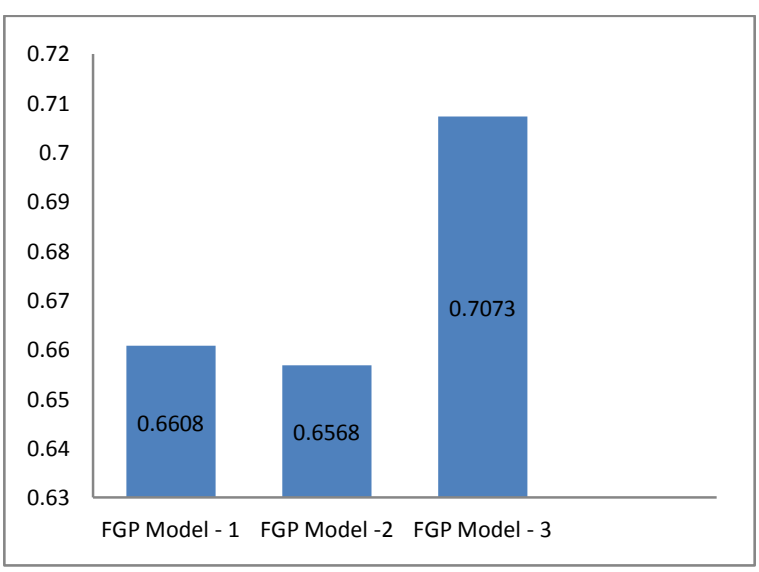

Figure 1. Distances obtained from three FGP models

Figure1 reflects that model 2 offers the best compromise solution for the problem 9.1.

\subsection{Example - 2}

Consider the following problem studied in [15]

$$
\begin{aligned}
& \underset{x_{1}}{\operatorname{aax}_{x_{2}}} F_{1}(x)=6 x_{1}+2 x_{2}+3 x_{3} \\
& \operatorname{Max}_{x_{3}} F_{2}(x)=5 x_{1}+6 x_{2}+3 x_{3} \\
& \left.\operatorname{Pr}_{3} \mathrm{a}_{11} x_{1}+a_{12} x_{2}+a_{13} x_{3} \leq 8\right] \geq 0.95 \\
& \operatorname{Pr}\left[x_{1}+x_{2}+x_{3} \leq b_{1}\right] \geq 0.05 \\
& \operatorname{Pr}\left[a_{21} x_{1}+a_{22} x_{2}+a_{23} x_{3} \geq b_{2}\right] \geq 0.90
\end{aligned}
$$

Here, $\mathrm{E}\left(\mathrm{a}_{11}\right)=1$, var $\left(\mathrm{a}_{11}\right)=5, \mathrm{E}\left(\mathrm{a}_{12}\right)=3$, var $\left(\mathrm{a}_{12}\right)=16$, $\mathrm{E}\left(\mathrm{a}_{13}\right)=9, \operatorname{var}\left(\mathrm{a}_{13}\right)=4, \mathrm{E}\left(\mathrm{b}_{1}\right)=2.5, \operatorname{var}\left(\mathrm{b}_{1}\right)=2, \mathrm{E}\left(\mathrm{a}_{21}\right)$ $=5, \operatorname{var}\left(\mathrm{a}_{21}\right)=3, \mathrm{E}\left(\mathrm{a}_{22}\right)=6$, var $\left(\mathrm{a}_{22}\right)=4, \mathrm{E}\left(\mathrm{a}_{23}\right)=8$, var $\left(\mathrm{a}_{23}\right)=5.5, \mathrm{E}\left(\mathrm{b}_{2}\right)=7, \operatorname{var}\left(\mathrm{b}_{2}\right)=5$.
In the table 3 the solution obtained from Kumar and Pal's method [15] and solution obtained from proposed method are compared using Euclidean distances (See Figure 2).

Table 3. Comparison between two methods

\begin{tabular}{|l|l|}
\hline $\begin{array}{l}\text { Kumar and Pal's Method } \\
{[15]}\end{array}$ & $\begin{array}{l}\text { Proposed Method (Model - } \\
1,2,3)\end{array}$ \\
\hline $\mathrm{x}_{1}=0.5075$ & $\mathrm{x}_{1}=1.567$ \\
\hline $\mathrm{x}_{2}=0.5929$ & $\mathrm{x}_{2}=0.05$ \\
\hline $\mathrm{x}_{3}=0$ & $\mathrm{x}_{3}=0.056$ \\
\hline $\mathrm{F}_{1}=5.1308$ & $\mathrm{~F}_{1}=9.67$ \\
\hline $\mathrm{F}_{2}=5.899$ & $\mathrm{~F}_{2}=7.703$ \\
\hline $\mathrm{F}_{3}=2.7937$ & $\mathrm{~F}_{3}=3.732$ \\
\hline$\mu_{\mathrm{F}_{1}}=0.9968$ & $\mu_{\mathrm{F}_{1}}=0.998$ \\
\hline$\mu_{\mathrm{F}_{2}}=0.7371$ & $\mu_{\mathrm{F}_{2}}=0.447$ \\
\hline$\mu_{\mathrm{F}_{3}}=0.2717$ & $\mu_{\mathrm{F}_{3}}=0.641$ \\
\hline$D=0.7743$ & $\mathrm{D}=0.6593$ \\
\hline
\end{tabular}

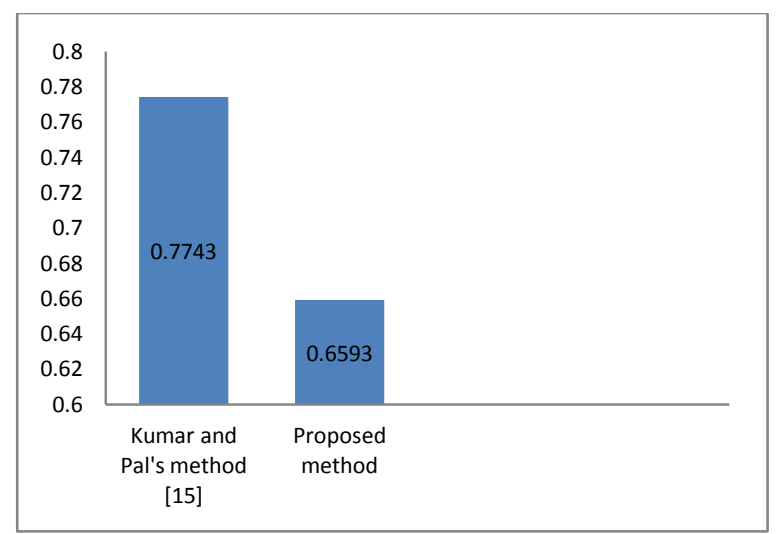

Figure 2. Comparison of Euclidean distance

Figure 2. reflects that proposed method provides the best compromise solution.

\section{ACKNOWLEDGMENTS}

The authors are very thankful to the referee for the valuable and constructive comments and suggestions.

\section{CONCLUSION}

Chance constrained multi - level linear programming problem is presented in this paper. Three types of chance constraints and its equivalent deterministic constraints are discussed. Each level decision maker provides his/her choice on bounds of the decision variable controlled by him/her. Three simple and comprehensive FGP models are used to solve the problem. Only under deviational variables are used in FGP models and no over deviational variables are needed to solve the problem. In other words, the problem is solved using fewer variables than the model used in [15]. It is also clear that the proposed FGP model offers better solution than the FGP model established in [15]. In the real world hierarchical 
decision making system, proposed approach is useful to make overall satisfactory decision in the decision making context.

The future scope of the mentioned idea is that this approach can be extended to multi objective multi - level linear programming with chance constraints, multi objective multi level linear decentralized programming with chance constraints. Further the proposed approach can be also extended to non -linear multi objective multi - level linear programming with chance constraints.

\section{REFERENCES}

[1] Anandalingam, G. 1988. A mathematical programming model of decentralized multi-level systems. Journal of the Operational Research Society 39 (11), 1021-1033.

[2] Anandalingam, G. and Apprey, V. 1991. Multilevel programming and conflicting resolution. European Journal of Operational Research 51, 233-247.

[3] Bard, J.F. and Falk, J.E. 1982. An explicit solution to the multi-level programming problems. Computers and Operations Research 9 (1), 77-100.

[4] Burton, R.M. 1977. The multilevel approach to organizational issues of the firm. Omega 5, 457-468.

[5] Lai, Y.J. 1996. Hierarchical optimization: a satisfactory solution. Fuzzy Sets and Systems 77, 321-335.

[6] Baky, I.A. 2010. Solving multi-level multi-objective linear programming problems through fuzzy goal programming approach. Applied Mathematical Modelling 34 (9), 2377-2387.

[7] Lachhwani, K. 2014. On solving multi-level multi objective linear programming problems through fuzzy goal programming approach. OPSEARCH 51(4), 624637.

[8] Shih, H.S., Lai, Y.J., and Lee, E.S. 1996. Fuzzy approach for multi-level programming problems. Computers \& Operations Research 23 (1), 73-91.

[9] Shih, H.S. and Lee, E.S. 2000. Compensatory fuzzy multiple level decision making. Fuzzy Sets and Systems 114 (1), 71-87.

[10] Sakawa, M., Nishizaki, I., and Uemura, Y. 1998. Interactive fuzzy programming for multilevel linear programming problems. Computers and Mathematics with Applications 36 (2), 71-86.

[11] Sakawa, M., Nishizaki, I., and Hitaka, M. 1999. Interactive fuzzy programming for multi-level 0-1 programming through genetic algorithms. European Journal of Operational Research 144(3), 580 - 588.
[12] Sinha, S. 2003. Fuzzy mathematical programming applied to multi-level programming problems. Computers and Operations Research 30 (9), 1259 - 1268.

[13] Sinha, S. 2003. Fuzzy programming approach to multilevel programming problems. Fuzzy Sets and Systems $136(2), 189-202$

[14] Pramanik, S. and Roy, T.K. 2007. Fuzzy goal programming approach to multi-level programming problem. European Journal of Operational Research 176(2), 1151-1166.

[15] Kumar, M. and Pal, B.B. 2013. Fuzzy Goal Programming Approach to Chance Constrained Multilevel Programming Problems. International Journal of Advanced Computer Research 3(8), 193-200.

[16] Charnes, A. and Cooper, W.W. 1959. Chanceconstrained programming. Management Science 6, 7379 .

[17] Emam O.E. and Nasr, S.A. 2014. A three-level quadratic programming problem with random rough coefficient in constraints. International Journal of Mathematical Archive 5(6), 11-18.

[18] Emam, O.E., El-Araby, M., and Belal, M.A. 2015. On Rough multi-level linear programming problem. Information Sciences Letters 4(1), 41-49.

[19] Pramanik, S. and Dey, P.P. 2011. Multi-objective quadratic programming problem based on fuzzy goal programming. International Journal of Pure and Applied Sciences and Technology 6(1), 45- 53.

[20] Pramanik, S. and Banerjee, D. 2012. Chance constrained quadratic bilevel programming problem. International Journal of Modern Engineering Research 2(4), 2417 2424.

[21] Pramanik, S., Banerjee, D., and Giri, B. C. 2012. Chance Constrained linear plus linear fractional bilevel programming problem. International Journal of Computer Applications 56(16), 34 - 39.

[22] Hulsurkar, S., Biswal, M.P., and Sinha, S.B. 1997. Fuzzy programming approach to multi-objective stochastic linear programming problems. Fuzzy Sets and Systems $88,173-181$.

[23] Pramanik, S. and Roy, T. K. 2008. Multiobjective transportation model with fuzzy parameters: based on priority based fuzzy goal programming approach. Journal of Transportation Systems Engineering and Information Technology 8(3), 40-48.

[24] Zeleny, M. 1982. Multiple criteria decision making. McGraw-Hill, New York. 